\title{
One-Dimensional Reaction-Diffusion Equations and Simple Source-Sink Arcs on a Circle
}

\author{
O. V. Pochinka, A.S. Loginova, E. V. Nozdrinova
}

This article presents a number of models that arise in physics, biology, chemistry, etc., described by a one-dimensional reaction-diffusion equation. The local dynamics of such models for various values of the parameters is described by a rough transformation of the circle. Accordingly, the control of such dynamics reduces to the consideration of a continuous family of maps of the circle. In this connection, the question of the possibility of joining two maps of the circle by an arc without bifurcation points naturally arises. In this paper it is shown that any orientation-preserving source-sink diffeomorphism on a circle is joined by such an arc. Note that such a result is not true for multidimensional spheres.

Keywords: reaction-diffusion equation, source-sink arc

\section{Introduction}

A wide range of models emerging in physics, biology, chemistry, etc. is described by the onedimensional reaction-diffusion equation. The corresponding chain of connected maps (CCM) or discrete variants of this equation can be regarded as an infinite set of copies of the local dynamical system on the phase space $\mathbb{R}$ or its compactification $\mathbb{S}^{1}$. If the local dynamics exhibits hyperbolic behavior, then the dynamics of the entire CCM is completely determined by local dynamics with sufficiently small parameters (see, for example, [5]).

So the Kolmogorov - Petrovsky - Piskunov (KPP) model of the development of useful genes is described by a local map $f(u)=u+\gamma u(1-u)$. For any $\gamma \in(0,1)$ the compactification map $\widetilde{f}$ is a Morse-Smale diffeomorphism of the circle $S^{1}$ with four fixed points (see [5, Theorem 5.1.1]).

Received May 30, 2018

Accepted June 23, 2018

This study was carried out within the framework of the RFBR project 18-31-00022 and the Basic Research Program at the National Research University Higher School of Economics (HSE) in 2018.

Olga V. Pochinka

olga-pochinka@yandex.ru

Anastasia S. Loginova

n.logi@mail.ru

Elena V. Nozdrinova

maati@mail.ru

National Research University Higher School of Economics (HSE)

ul. B. Pecherskaya 25/12, Nizhny Novgorod, 603150, Russia

RUSSIAN JOURNAL OF NONLINEAR DYNAMICS, 2018, 14(3), 325-330 
The Nagumo model of propagation of a voltage pulse through the neuron axon is described by a local map $f(u)=u-A u(u-\Theta)(u-1)$. For all sufficiently small $A$ the compactification map $\tilde{f}$ is a Morse-Smale diffeomorphism of the circle $S^{1}$ with four fixed points (see [5, Theorem 5.1.1]).

The real amplitude equation of waves generated by the wind is described by a local map $f(u)=A u-b u^{3}$. If $b>0$ and $A \in(0,1) \cup\left(1, \frac{3}{2}\right)$, then the compactifying map $\widetilde{f}$ is the MorseSmale diffeomorphism of the circle $S^{1}$ with four fixed points at $A \in\left(1, \frac{3}{2}\right)$ and two fixed points at $A \in(0,1)$ (see [5, Theorem 5.3.1]).

The control of the dynamics of the described models reduces to the consideration of a continuous family of maps of the circle. This raises the question of the possibility of joining two maps of the circle by an arc without bifurcation points. In this paper we show that such an arc joins any orientation-preserving source-sink diffeomorphisms on the circle. In more detail.

Let us consider the circle $\mathbb{S}^{1}=\left\{\left(x_{1}, x_{2}\right) \in \mathbb{R}^{2}: x_{1}^{2}+x_{2}^{2}=1\right\}$. We denote by $\operatorname{Diff}\left(\mathbb{S}^{1}\right)$ a set of diffeomorphisms on a given circle and by $J\left(\mathbb{S}^{1}\right) \subset \operatorname{Diff}\left(\mathbb{S}^{1}\right)$ a set of orientation-preserving source-sink diffeomorphisms, i.e., diffeomorphisms whose nonwandering sets consist of two hyperbolic points: a source and a sink.

Theorem 1. For any diffeomorphisms $f, f^{\prime} \in J\left(\mathbb{S}^{1}\right)$ there is a smooth arc $\left\{f_{t} \in J\left(\mathbb{S}^{1}\right)\right\}$ joining them.

We note that the multidimensional reaction-diffusion equations reduce to a source-sink diffeomorphism on a multidimensional sphere for which the result of Theorem 1 is not true (see, for example, [2]).

\section{Supporting concepts and facts}

Two diffeomorphisms $f, f^{\prime}: \mathbb{S}^{1} \rightarrow \mathbb{S}^{1}$ are smoothly isotopic if there is a smooth map $F: \mathbb{S}^{1} \times[0,1] \rightarrow \mathbb{S}^{1}$ (smooth isotopy) such that $f_{t}$ given by the formula $f_{t}(x)=f(x, t)$ is a diffeomorphism for each $t \in[0,1]$ and $f_{0}=f, f_{1}=f^{\prime}$. We say that the family $\left\{f_{t}\right\}$ is a smooth arc joining diffeomorphisms $f$ and $f^{\prime}$. A support of the isotopy $\left\{f_{t}\right\}$ is the closure of the set $\left\{x \in \mathbb{S}^{1}: f_{t}(x) \neq f_{0}(x)\right.$ for some $\left.t \in[0,1]\right\}$.

For the smooth arcs $\left\{f_{t}\right\}$ and $\left\{g_{t}\right\}$ such that $f_{1}=g_{0}$ the usually product of the respective paths is not smooth in general, but we can define the smooth arc $\left\{h_{t}\right\}$ joining diffeomorphisms $f_{0}, g_{1}$ as follows:

$$
h_{t}= \begin{cases}f_{2 \tau(t)}, & 0 \leqslant t \leqslant \frac{1}{2}, \\ g_{2 \tau(t)-1}, & \frac{1}{2} \leqslant t \leqslant 1,\end{cases}
$$

where $\tau:[0,1] \rightarrow[0,1]$ is a smooth monotone function such that $\tau(t)=0$ for $0 \leqslant t \leqslant \frac{1}{3}$ and $\tau(t)=1$ for $\frac{2}{3} \leqslant t \leqslant 1$. We will call the smooth arc $\left\{h_{t}\right\}$ a smooth product of the smooth $\operatorname{arcs}\left\{f_{t}\right\}$ and $\left\{g_{t}\right\}$ and denote it by $\left\{h_{t}\right\}=\left\{f_{t} * g_{t}\right\}$.

In the present paper we will work with the class $J\left(\mathbb{S}^{1}\right) \subset \operatorname{Diff}\left(\mathbb{S}^{1}\right)$ of orientation-preserving source-sink diffeomorphisms, that is, diffeomorphisms whose nonwandering sets consist of precisely two hyperbolic points: a source and a sink. Also, we introduce a subclass $N S\left(\mathbb{S}^{1}\right) \subset J\left(\mathbb{S}^{1}\right)$ for which the source is the North pole $N(0,1)$ and the sink is the South pole $S(0,-1)$. 
Define the diffeomorphisms

$$
\begin{aligned}
& \vartheta_{-}: \mathbb{S}^{1} \backslash\{S\} \rightarrow \mathbb{R}, \\
& \vartheta_{+}: \mathbb{S}^{1} \backslash\{N\} \rightarrow \mathbb{R}
\end{aligned}
$$

(the stereographic projections) by the formulas:

$$
\begin{aligned}
& \vartheta_{-}\left(x_{1}, x_{2}\right)=\frac{x_{1}}{1+x_{2}}, \\
& \vartheta_{+}\left(x_{1}, x_{2}\right)=\frac{x_{1}}{1-x_{2}} .
\end{aligned}
$$

Define the diffeomorphisms

$$
\begin{aligned}
& \vartheta_{-}^{-1}: \mathbb{R} \rightarrow \mathbb{S}^{1} \backslash\{S\} \\
& \vartheta_{+}^{-1}: \mathbb{R} \rightarrow \mathbb{S}^{1} \backslash\{N\}
\end{aligned}
$$

which are given by the formulas:

$$
\begin{aligned}
& \vartheta_{-}^{-1}(x)=\left(\frac{2 x}{x^{2}+1}, \frac{1-x^{2}}{x^{2}+1}\right), \\
& \vartheta_{+}^{-1}(x)=\left(\frac{2 x}{x^{2}+1}, \frac{x^{2}-1}{x^{2}+1}\right) .
\end{aligned}
$$

Through stereographic projections, diffeomorphisms from $N S\left(\mathbb{S}^{1}\right)$ are associated with the contractions on the line

$$
S(\mathbb{R})=\left\{\vartheta_{+} f \vartheta_{+}^{-1}, f \in N S\left(\mathbb{S}^{1}\right)\right\}
$$

and extensions on the line

$$
N(\mathbb{R})=\left\{\vartheta_{-} f \vartheta_{-}^{-1}, f \in N S\left(\mathbb{S}^{1}\right)\right\}
$$

We define model diffeomorphisms $g: \mathbb{S}^{1} \rightarrow \mathbb{S}^{1}, \bar{g}: \mathbb{R} \rightarrow \mathbb{R}$ by the formulas

$$
\begin{aligned}
g\left(x_{1}, x_{2}\right) & =\left(\frac{4 x_{1}}{5-3 x_{2}}, \frac{5 x_{2}-3}{5-3 x_{2}}\right), \\
\bar{g}(x) & =\left(\frac{x}{2}\right) .
\end{aligned}
$$

It is easy to verify that $g \in N S\left(\mathbb{S}^{1}\right), \bar{g} \in S(\mathbb{R}), \bar{g}^{-1} \in N(\mathbb{R}), \bar{g}^{-1}=\vartheta_{-} g \vartheta_{-}^{-1}$ and $\bar{g}=\vartheta_{+} g \vartheta_{+}^{-1}$.

Let $E_{g} \subset N S\left(\mathbb{S}^{1}\right)$ be a set of such diffeomorphisms $h$ for which there are neighborhoods $V_{h}(N), V_{h}(S)$ of points $N, S$ such that $\left.h\right|_{V_{h}(N) \cup V_{h}(S)}=\left.g\right|_{V_{h}(N) \cup V_{h}(S)}$. We denote by $E_{\bar{g}} \subset S(\mathbb{R})\left(E_{\bar{g}^{-1}} \subset N(\mathbb{R})\right)$ a set of diffeomorphisms $\bar{h}$ for which there is a neighborhood $V_{\bar{h}}(O)$ of the point $O$ such that $\left.\bar{h}\right|_{V_{\bar{h}}(O)}=\left.\bar{g}\right|_{V_{\bar{h}}(O)}\left(\left.\bar{h}\right|_{V_{\bar{h}}(O)}=\left.\bar{g}^{-1}\right|_{V_{\bar{h}}(O)}\right)$.

\section{The construction of a smooth arc is a proof of Theorem 1}

It is easy to prove Theorem 1 if $f$ and $f^{\prime}$ smoothly conjugate by a diffeomorphism $h$. In this case there is a smooth isotopy $h_{t}: \mathbb{S}^{1} \rightarrow \mathbb{S}^{1}$ such that $h_{0}=i d, h_{1}=h$. Thus, $f_{t}=h_{t}^{-1} f h_{t}$ is a required arc. In the opposite case we show how to construct a smooth $\operatorname{arc}\left\{l_{t} \in J\left(\mathbb{S}^{1}\right)\right\}$ joining an arbitrary diffeomorphism $f \in J\left(\mathbb{S}^{1}\right)$ with the model diffeomorphism $g$. Similarly, 
we construct a smooth $\operatorname{arc}\left\{l_{t}^{\prime} \in J\left(\mathbb{S}^{1}\right)\right\}$ joining $f^{\prime}$ and $g$. Then the smooth product of smooth $\operatorname{arcs} f_{t}=l_{t} * l_{1-t}^{\prime}$ is the required arc. The arc $l_{t}$ is a smooth product of tree smooth $\operatorname{arcs} \beta_{t}, \gamma_{t}, \delta_{t}$ $\left(l_{t}=\beta_{t} * \gamma_{t} * \delta_{t}\right)$, whose construction is given in the corresponding sentences below. Thus,

1. $l_{t}$ is a diffeomorphism from the class $J\left(\mathbb{S}^{1}\right)$ for all $t \in\left[0, \frac{1}{3}\right], l_{0}=f$ and $l_{\frac{1}{3}}$ is a diffeomorphism from the class $N S\left(\mathbb{S}^{1}\right)$ (see Proposition 1);

2. $l_{t}$ is a diffeomorphism from the class $N S\left(\mathbb{S}^{1}\right)$ for all $t \in\left[\frac{1}{3}, \frac{2}{3}\right], l_{\frac{2}{3}} \in E_{g}$ (see Proposition 2);

3. $l_{t}$ is a diffeomorphism from the class $E_{g}$ for all $t \in\left[\frac{2}{3}, 1\right], l_{1}=g$ (see Proposition 3 ).

Proposition 1. For any diffeomorphism $f \in J\left(\mathbb{S}^{1}\right)$ there exists a smooth arc $\left\{\beta_{t} \in\left(J\left(\mathbb{S}^{1}\right) \cap \operatorname{Diff}\left(\mathbb{S}^{1}\right)\right)\right\}$ joining $\beta_{0}=f$ with a diffeomorphism $\beta_{1} \in N S\left(\mathbb{S}^{1}\right)$.

Proof. Let $f \in J\left(\mathbb{S}^{1}\right)$. We denote by $\alpha$ the source and by $\omega$ the sink of the diffeomorphism $f$. Let $D_{\alpha}, D_{\omega}\left(D_{S}, D_{N}\right)$ be pairwise disjoint arcs of a circle containing $\alpha, \omega(S, N)$. According to [3], there exists a smooth arc $\left\{H_{t} \in \operatorname{Diff}\left(\mathbb{S}^{1}\right)\right\}$ such that $H_{0}=i d, H_{1}\left(D_{N}\right)=D_{\alpha}$, $H_{1}\left(D_{S}\right)=D_{\omega}, H_{1}(N)=\alpha$ and $H_{1}(S)=\omega$. Then $\beta_{t}=H_{t}^{-1} f H_{t}$ is the desired isotopy joining the diffeomorphism $f=\beta_{0}$ with the diffeomorphism $\beta_{1}=H_{1}^{-1} f H_{1} \in N S\left(\mathbb{S}^{1}\right)$.

Proposition 2. For any diffeomorphism $\beta \in N S\left(\mathbb{S}^{1}\right)$ there exists a smooth arc $\left\{\gamma_{t} \in N S\left(\mathbb{S}^{1}\right)\right\}$ joining $\gamma_{0}=\beta$ with a diffeomorphism $\gamma_{1} \in E_{g}$.

Proof. Define $\bar{\beta}^{+}=\vartheta_{+} \beta \vartheta_{+}{ }^{-1}$ and $\bar{\beta}^{-}=\vartheta_{-} \beta \vartheta_{-}{ }^{-1}$. Then $\bar{\beta}^{+} \in S(\mathbb{R})$ and $\bar{\beta}^{-} \in N(\mathbb{R})$. If $\bar{\beta}^{+}=\bar{g}$ in some neighborhood of the origin $O$, then we define $\left\{\bar{\gamma}_{t}^{+}=\bar{\beta}^{+}\right\}$. In the opposite case we show below how to construct a smooth $\operatorname{arc}\left\{\bar{\gamma}_{t}^{+} \in S(\mathbb{R})\right\}$ joining $\bar{\beta}^{+}$with a diffeomorphism $\bar{\gamma}_{1}^{+} \in E_{\bar{g}}$ such that $\bar{\gamma}_{t}^{+}$for every $t \in[0,1]$ coincides with $\bar{\beta}^{+}$out of a neighborhood $V^{+} \subset \mathbb{B}^{1}=$ $=\{x \in \mathbb{R}:|x| \leqslant 1\}$ of the origin $O$. Similarly, if $\bar{\beta}^{-}=\bar{g}^{-1}$ in some neighborhood of the origin $O$, then we define $\left\{\bar{\gamma}_{t}^{-}=\bar{\beta}^{-}\right\}$. In the opposite case, similarly to $\bar{\gamma}_{t}^{+}$we construct a smooth arc $\left\{\bar{\gamma}_{t}^{-} \in N(\mathbb{R})\right\}$ joining $\bar{\beta}^{-}$with a diffeomorphism $\bar{\gamma}_{1}^{-} \in E_{\bar{g}^{-1}}$ such that $\bar{\gamma}_{t}^{-}$for every $t \in[0,1]$ coincides with $\bar{\beta}^{-}$out of a neighborhood $V^{-} \subset \mathbb{B}^{1}$ of the origin $O$. Thus, the required arc $\gamma_{t}$ given by the formula

$$
\gamma_{t}(w)=\left\{\begin{array}{l}
\vartheta_{+}{ }^{-1}\left(\gamma_{t}^{+}\left(\vartheta_{+}(w)\right)\right), \quad w \in \vartheta_{+}{ }^{-1}\left(V^{+}\right) ; \\
\vartheta_{-}^{-1}\left(\gamma_{t}^{-}\left(\vartheta_{-}(w)\right)\right), \quad w \in \vartheta_{-}^{-1}\left(V^{-}\right) ; \\
\beta(w), w \in\left(\mathbb{S}^{1} \backslash\left(\vartheta_{+}{ }^{-1}\left(V^{+}\right) \cup \vartheta_{-}^{-1}\left(V^{-}\right)\right) .\right.
\end{array}\right.
$$

Let us construct a smooth arc $\left\{\bar{\gamma}_{t}^{+} \in S(\mathbb{R})\right\}$ joining $\bar{\beta}^{+}$with a diffeomorphism $\bar{\gamma}_{1}^{+} \in E_{\bar{g}}$ such that $\bar{\gamma}_{t}^{+}$for every $t \in[0,1]$ coincides with $\bar{\beta}^{+}$out of a neighborhood $V^{+} \subset \mathbb{B}^{1}$. The arc $\bar{\gamma}_{t}^{+}$ will be a smooth product of arcs: $\bar{\rho}_{t}^{+}$and $\bar{\xi}_{t}^{+}$, where

1) $\left\{\bar{\rho}_{t}^{+} \in S(\mathbb{R})\right\}$ joining the diffeomorphism $\bar{\beta}^{+}$with a diffeomorphism $\bar{\rho}_{1}^{+}$such that $\bar{\rho}_{t}^{+}$ for every $t \in[0,1]$ coincides with $\bar{\beta}^{+}$out of a neighborhood $V_{1}^{+} \subset \mathbb{B}^{1}$ of the origin $O$ and $\bar{\rho}_{1}^{+}$ coincides with differential $D \bar{\beta}_{O}^{+}$(denote it by $Q$ ) in a neighborhood $V_{2}^{+} \subset V_{1}^{+}$of the origin $O$;

2) $\left\{\bar{\xi}_{t}^{+} \in S(\mathbb{R})\right\}$ joining the diffeomorphism $\bar{\rho}_{1}^{+}$with a diffeomorphism $\bar{\xi}_{1}^{+} \in E_{\bar{g}}$ such that $\bar{\xi}_{t}^{+}$ for every $t \in[0,1]$ coincides with $\bar{\rho}_{1}^{+}$out of a neighborhood $V_{3}^{+} \subset V_{2}^{+}$. 
1) Since $O$ is a hyperbolic sink for diffeomorphism $\bar{\beta}^{+}$, it follows that

$$
Q(x)=\lambda x
$$

for some $0<\lambda<1$. Since the map $\bar{\beta}^{+}$in some neighborhood of the origin has the form $\bar{\beta}^{+}(x)=x(\lambda+a(x))$ where $a(x)$ tends to 0 with $x$ tending to 0 , there exists a number $\ell_{1}>0$ such that in the neighborhood $U_{1}^{+}=\left\{x \in \mathbb{R}:|x| \leqslant \ell_{1}\right\}$ of the point $O$ for all $x \neq 0$ there is the inequality $\left|\bar{\beta}^{+}(x)\right|<|x|$. Define the arc $\bar{\chi}_{t}^{+}: \mathbb{R} \rightarrow \mathbb{R}$ by the formula

$$
\bar{\chi}_{t}^{+}=(1-t) \bar{\beta}^{+}+t Q .
$$

Then $\left|\bar{\chi}_{t}^{+}(x)\right|<|x|$ for all nonzero $x \in U_{1}^{+}$. Note that the origin $O$ is a fixed point for each diffeomorphism $\bar{\chi}_{t}^{+}$and $\bar{\chi}_{t}^{+}\left(U_{1}^{+}\right) \subset$ int $U_{1}^{+}$for every $t \in[0,1]$. Let $V_{+}^{1}=\left(\bar{\beta}^{+}\right)^{-1}\left(U_{1}^{+}\right)$and let us consider the isotopy $h_{t}=\left(\bar{\beta}^{+}\right)^{-1} \bar{\chi}_{t}^{+}$, which connects the identity map with the diffeomorphism $\left(\bar{\beta}^{+}\right)^{-1} Q$. By construction $h_{t}\left(U_{1}^{+}\right) \subset V_{1}^{+}$for every $t \in[0,1]$. According to Thom's theorem about the continuation of an isotopy (see, for example, [4, Theorem 5.8]), there is an isotopy $H_{t}: \mathbb{R} \rightarrow \mathbb{R}$, which coincides with $h_{t}$ on $U_{1}^{+}$and is the identity outside $V_{1}^{+}$. Then the required arc is given by

$$
\bar{\rho}_{t}^{+}=\bar{\beta}^{+} H_{t}
$$

2) If $Q=\bar{g}$, then we define $\left\{\bar{\xi}_{t}^{+}=\bar{\rho}_{1}^{+}\right\}$. In the opposite case we define the arc $\bar{\tau}_{t}^{+}: \mathbb{R} \rightarrow \mathbb{R}$ by the formula

$$
\bar{\tau}_{t}^{+}=(1-t) Q+t \bar{g}
$$

Let $V_{2}^{+}=U_{1}^{+}$and $U_{2}^{+}=Q\left(U_{1}^{+}\right)$. Consider the isotopy $\eta_{t}=Q^{-1} \bar{\tau}_{t}^{+}$which connects the identity map with the diffeomorphism $Q^{-1} \bar{g}$. By the construction $\eta_{t}\left(U_{2}^{+}\right) \subset V_{2}^{+}$for every $t \in[0,1]$. According to Thom's theorem about the continuation of an isotopy (see, for example, [4, Theorem 5.8]), there is an isotopy $\theta_{t}: \mathbb{R} \rightarrow \mathbb{R}$, which on $U_{2}^{+}$is $\theta_{t}$ and which is the identity outside $V_{2}^{+}$. Then the required arc is given by

$$
\bar{\xi}_{t}^{+}=\bar{\rho}_{1}^{+} \theta_{t} .
$$

Proposition 3. For any diffeomorphism $\gamma \in E_{g}$ there is a smooth arc $\left\{\delta_{t} \in J\left(\mathbb{S}^{1}\right)\right\}$ joining $\delta_{0}=\gamma$ with $\delta_{1}=g$.

Proof. Since $\gamma \in E_{g}$, there are neighborhoods $V_{\gamma}(N)$ and $V_{\gamma}(S)$ of the points $N$ and $S$ such that $\left.\gamma\right|_{V_{\gamma}(N) \cup V_{\gamma}(S)}=\left.g\right|_{V_{\gamma}(N) \cup V_{\gamma}(S)}$. We define a diffeomorphism $\psi_{\gamma}: \mathbb{S}^{1} \backslash\{S\} \rightarrow \mathbb{S}^{1} \backslash\{S\}$ by the formula $\psi_{\gamma}(w)=g^{k}\left(\gamma^{-k}(w)\right)$ where $k \in \mathbb{Z}$ such that $\gamma^{-k}(w) \in V_{\gamma}(N)$ for $w \in \mathbb{S}^{1} \backslash\{S\}$. Then $\gamma=\psi_{\gamma}^{-1} g \psi_{\gamma}$. If $\psi_{\gamma}$ can be continued smoothly on $S$ by the formula $\psi_{\gamma}(S)=S$, then there exists a smooth isotopy $\rho_{t}$ : $\mathbb{S}^{1} \rightarrow \mathbb{S}^{1}$, such that $\rho_{0}=\psi, \rho_{1}=i d$. Thus, the arc $\delta_{t}=\rho_{t}^{-1} g \rho_{t}$ is the desired arc. In the opposite case we show that there exists a smooth arc $\left\{\zeta_{t} \in E_{g}\right\}$ joining the diffeomorphism $\zeta_{0}=\gamma$ with some diffeomorphism $\zeta_{1}$, such that $\psi_{\zeta_{1}}$ can be continued smoothly on $\mathbb{S}^{1}$ by the formula $\psi_{\zeta_{1}}(S)=S$.

Let $\mathbb{B}_{r}=\{x \in \mathbb{R}:|x| \leqslant r\}$ and $K_{r}=c l\left(\mathbb{B}_{r} \backslash \mathbb{B}_{\frac{r}{2}}\right)$ for $r>0$. Let $\bar{\gamma}=\vartheta_{+} \gamma \vartheta_{+}{ }^{-1}$ and $\bar{\psi}_{\gamma}=\vartheta_{+} \psi_{\gamma} \vartheta_{+}{ }^{-1}$. Then $\bar{\gamma} \in E_{\bar{g}}$ and, therefore, there is $r_{0}>0$ such that $\bar{\gamma}=\bar{g}$ on $\mathbb{B}_{r_{0}}$ and the space $K_{r_{0}}$ is a fundamental domain of the restriction of the diffeomorphism $\bar{g}$ (as well as the diffeomorphism $\bar{\gamma})$ on $\mathbb{R} \backslash\{O\}$. By construction, the orbit space $C=(\mathbb{R} \backslash\{O\}) / \bar{g}$ is a disjoint union of two circles $C=C_{+} \cup C_{-}$. We denote by $p: \mathbb{B}_{r_{0}} \backslash\{O\} \rightarrow C$ the natural projection. 
Then the curves $a_{+}=p\left(O x^{+}\right), a_{-}=p\left(O x^{-}\right)$are generators of fundamental groups $\pi_{1}\left(C_{+}\right)$ and $\pi_{1}\left(C_{-}\right)$, respectively.

Since $\bar{\psi}_{\gamma}$ translates orbits of the diffeomorphism $\bar{g}$ into orbits of the diffeomorphism $\bar{\gamma}$ and $K_{r_{0}}$ is a common fundamental area for $\bar{g}, \bar{\gamma}$ on $\mathbb{R} \backslash\{O\}$, it follows that $\bar{\psi}_{\gamma}$ is designed for $C_{ \pm}$by the diffeomorphism $\widehat{\psi}_{\gamma_{ \pm}}=p \bar{\psi}_{\gamma} p^{-1}: C_{ \pm} \rightarrow C_{ \pm}$. Moreover, the induced isomorphism $\widehat{\psi}_{\gamma_{ \pm} *}: \pi_{1}\left(C_{ \pm}\right) \rightarrow \pi_{1}\left(C_{ \pm}\right)$is the identity. Thus, the diffeomorphism $\widehat{\psi}_{\gamma_{ \pm}}$is smoothly isotopic to the identity map.

We choose an open covering $U^{ \pm}=\left\{U_{1}^{ \pm}, \ldots, U_{q}^{ \pm}\right\}$of the manifold $C_{ \pm}$such that each connected component of the set $p^{-1}\left(U_{i}^{ \pm}\right)$is a subset of $K_{r_{i}}$ for some $r_{i}<\frac{r_{i-1}}{2}$. According to the fragmentation lemma (see, for example, [1]), there exist diffeomorphisms $\widehat{w}_{1}^{ \pm}, \ldots, \widehat{w}_{q}^{ \pm}: C_{ \pm} \rightarrow C_{ \pm}$ such that they are smoothly isotopic to the identity map and

i) for each $i=\overline{1, q}$ and for each $t \in[0,1]$ diffeomorphism $\widehat{w}_{i, t}^{ \pm}$is the identity outside $U_{i}^{ \pm}$ and $\left\{\widehat{w}_{i, t}^{ \pm}\right\}$is the smooth isotopy between the identity map and $\widehat{w}_{i}^{ \pm}$;

ii) $\widehat{\psi}_{\gamma_{ \pm}}=\widehat{w}_{1}^{ \pm} \ldots \widehat{w}_{q}^{ \pm}$.

Let $\bar{w}_{i, t}^{ \pm}: \mathbb{R} \rightarrow \mathbb{R}$ be a diffeomorphism coinciding with $\left(\left.p\right|_{K_{r_{i}}}\right)^{-1} \widehat{w}_{i, t}^{ \pm} p$ on $K_{r_{i}}$ and coin-

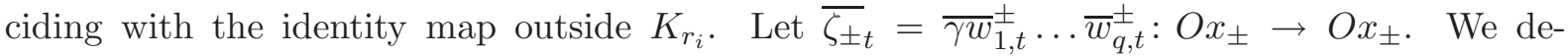
note by $\bar{\zeta}_{t}: \mathbb{R} \rightarrow \mathbb{R}$ a diffeomorphism coinciding with $\bar{\zeta}+t_{t}$ on $O x_{+}$, with $\bar{\zeta}_{-t}$ on $O x_{-}$and such that $\bar{\zeta}_{t}(O)=O$. By construction $\bar{\zeta}_{t} \in E_{\bar{g}}$ for every $t \in[0,1], \bar{\zeta}_{0}=\bar{\gamma}$ and $\widehat{\psi}_{\zeta_{1}}=i d$. Thus, $\psi_{\zeta_{1}}=\vartheta_{+}{ }^{-1} \bar{\psi}_{\zeta_{1}} \vartheta_{+}: \mathbb{S}^{1} \backslash\{S\} \rightarrow \mathbb{S}^{1} \backslash\{S\}$ can be continued smoothly on $\mathbb{S}^{1}$ by the formula $\psi_{\zeta_{1}}(S)=S$.

\section{References}

[1] Banyaga, A., On the Structure of the Group of Equivariant Diffeomorphisms, Topology, 1977, vol. 16, no. 3, pp. 279-283.

[2] Bonatti, C., Grines, V.Z., Medvedev, V.S., and Pochinka, O. V., Bifurcations of Morse-Smale Diffeomorphisms with Wildly Embedded Separatrices, Proc. Steklov Inst. Math., 2007, vol. 256, no. 1, pp. 47-61; see also: Tr. Mat. Inst. Steklova, 2007, vol. 256, pp. 54-69.

[3] Hirsch, M. W., Differential Topology, Grad. Texts in Math., vol. 33, New York: Springer, 1976.

[4] Milnor, J., Lectures on the h-Cobordism Theorem, Princeton, N.J.: Princeton Univ. Press, 1965.

[5] Pesin, Ya. B. and Yurchenko, A. A., Some Physical Models Described by the Reaction-Diffusion Equation, and Coupled Map Lattices, Russian Math. Surveys, 2004, vol.59, no. 3, pp. 481-513; see also: Uspekhi Mat. Nauk, 2004, vol. 59, no.3(357), pp. 81-114. 